\title{
A importância dos profissionais da saúde bucal no programa Consultório na Rua: Revisão Narrativa
}

\author{
The Importance of Oral Health Professionals in the Street Clinic program: Narrative \\ Review
}

\section{La importancia de los profesionales de la salud bucal en el programa Consultorio en la Calle: Revisión Narrativa}

Maria Izabel de Mendonça Alves ${ }^{1 *}$, Gabriel Lobo Gomes Vitorino ${ }^{2}$, Thaysa Priscilla Lacerda Liberato².

\section{RESUMO}

Objetivo: Descrever a importância dos profissionais da saúde bucal no programa Consultório na Rua. Revisão de literatura: Os moradores de rua encontram-se em situação de extrema vulnerabilidade. A violência é comumente observada no cotidiano dessas pessoas. Muitos relatam atitudes como a discriminação e negação de direitos, por parte da polícia e outras instituições públicas. A pesquisa nacional sobre a população em situação de rua, foi realizada em 71 cidades, e contou com a avaliação de 31922 pessoas adultas em vivendo nessa condição. Foi verificado que a principal circunstância que leva a essa situação foi o consumo abusivo de drogas. Nesse contexto, o governo brasileiro implementou o programa Consultório na Rua, que visa atender especificamente esses pacientes. A odontologia conseguiu ganhar espaço e se inseriu no quadro de atendimentos do programa Consultórios na Rua. Considerações finais: $O$ trabalho dos profissionais da saúde bucal no programa é o de gerar uma maior acessibilidade da odontologia a esse público-alvo.

Palavras-chave: Saúde bucal, Pessoas em situação de rua, Transtornos relacionados ao uso de substâncias.

\begin{abstract}
Goal: To describe the importance of the oral health professionals on the Street's Clinic program. Literature review: The homeless people are extremely vulnerable, and the violence is commonly observed in their daily lives. They report attitudes such as discrimination and denial of rights by police and other public institutions. A national survey with homeless people was conducted in 71 cities and found that 31922 adults are living in this condition. The result of the survey found that the main circumstance that lead these people to this situation is the abuse of narcotic substances. In this context, the Brazilian government has implemented the Street's Clinic program, whose goal is to serve these patients and consequently the dentistry was inserted on this governmental program. Final considerations: The work of the oral health professionals on the program is to promote greater accessibility of dentistry to this target audience.
\end{abstract}

Key words: Oral health, Homeless people, Substance Use Disorders.

${ }^{1}$ Universidade Federal de Alagoas (UFAL/FAMED). Maceió-Alagoas.

${ }^{2}$ Centro Universitário Cesmac (CESMAC), Maceió-Alagoas. *E-mail: gabriellobovitorino@hotmail.com

SUBMETIDO EM: $8 / 2019$

ACEITO EM: 9/2019

PUBLICADO EM: 11/2019 


\section{RESUMEN}

Objetivo: Describir la importancia de los profesionales de la salud bucal en el programa Consultorio en la Calle. Revisión de literatura: Las personas sin hogar son extremadamente vulnerables. La violencia se observa comúnmente en su vida diaria. Muchos informan actitudes como la discriminación y la negación de derechos por parte de la policía y otras instituciones públicas. La encuesta nacional sobre la población sin hogar se realizó en 71 ciudades y contó con 31922 adultos que viven en esta condición. Se encontró que la principal circunstancia que condujo a esta situación fue el abuso de drogas. En este contexto, el gobierno brasileño ha implementado el programa Consultorio en la Calle, cuyo objetivo es tratar específicamente a estos pacientes. La odontología pudo ganar espacio y se insertó en el servicio del programa Consultorio en la Calle. Consideraciones finales: El trabajo de los profesionales de la salud oral en el programa es generar una mayor accesibilidad de la odontología a este público objetivo.

Palabra chave: Salud bucal, Personas sin hogar, Trastornos de uso de sustancias.

\section{INTRODUÇÃO}

Morar na rua é uma batalha diária contra a fome, o vício, a natureza, a violência e a discriminação. Sobreviventes é um termo que os definem, pois apesar de estarem submetidos a condições extremas, continuam lutando com as forças que lhes restam para se manterem vivos (MORERA JAC e PADILHA MI, 2015).

A pesquisa nacional sobre a população em situação de rua, foi realizada em 71 cidades, e contou com a avaliação de 31922 pessoas adultas em condição de rua. Foi verificado que $82 \%$ dessas pessoas eram homens, $67 \%$ eram pessoas negras, e os principais circunstâncias que levaram a essa condição foram 0 consumo de drogas lícitas e ilícitas 35,5\%, desemprego 29,8\% e conflitos familiares 29,1\% (BRASIL, 2009).

Os moradores de rua encontram-se em situação de extrema vulnerabilidade. Violência física e moral é comumente observada no cotidiano dessas pessoas. Muitos em situação de rua relatam atitudes como o abuso do poder, discriminação e negação de direitos, por parte da polícia e outras instituições públicas (NONATO DM e RAIOL RWG, 2018).

Patologias como Vírus da Imunodeficiência Humana (HIV), hipertensão, problemas mentais, tuberculose, sífilis podem estar associadas a essa condição. Muitos desses moradores de rua procuram inicialmente os hospitais já em situações de urgência, não tendo contato com meios ou políticas de saúde preventivas até aquele momento (MINISTERIO DA SAÚDE, 2014).

Devido à falta de cuidados bucais e uso de drogas constante alterações bucais são comumente observadas nesses pacientes como gengivites, periodontites, perda precoce de dentes, cáries extensas, lesões em área de mucosa (MARQUES LARV, et al., 2015).

As pessoas que vivem em situação de rua não são homogêneas, possuem pensamentos, temperamento, costumes, crenças, e problemas diferentes. Tratar indivíduos de maneira igual por estarem inseridos dentro de uma mesma realidade social, pode gerar resultados não efetivos quando tratar-se do processo saúdedoença (PAIVA IKSD, et al., 2015).

Para atender especificamente as pessoas que estão na condição de moradores de rua, e que se em encontram na situação de dependência química, o governo brasileiro elaborou e implementou o projeto Consultórios na Rua, que atualmente são regulados pela portaria $\mathrm{n} . \stackrel{0}{122}$, de 25 de janeiro de 2011 , com a finalidade de prestar um serviço público de saúde mais efetivo para aquela categoria tão específica de necessitados, que se encontram em uma situação maior de vulnerabilidade (BRASIL, 2011).

Nesta perspectiva, o presente trabalho tem como objetivo fazer um apanhando teórico sobre a importância da atuação dos profissionais da saúde bucal que laboram promovendo nos consultórios de rua, o acolhimento e o acompanhando da população que se encontra nas ruas em situação de dependência química. 


\section{REVISÃO DE LITERATURA}

\section{Dependência química associada a alterações bucais}

Atualmente o abuso no consumo de substâncias psicoativas tornou-se um problema de saúde pública em todo o mundo (CRIME UNOD, 2018). Segundo dados da United Nations Office on Drugs and Crime (2018), no ano de 2016 estimou-se que cerca de 275 milhões de pessoas, com idade de 15 a 64 anos, utilizaram algum tipo de droga seja lícita ou ilícita.

Dentre as substancias permitidas para consumo pela lei brasileira temos o álcool que é atualmente a mais consumida no mundo, cigarros, charutos entre outras. Em relação as drogas ilícitas coibidas pela lei brasileira temos a maconha que é a droga ilícita mais utilizada no mundo, e em segundo lugar temos a cocaína (CRIME UNOD, 2018).

As drogas psicoativas ou psicotrópicas são substâncias que atuam no sistema nervoso central, e possuem capacidade de alterar as funções psicológicas, orgânicas e comportamentais dos usuários (CARLINE E, et al., 2015).

O consumo constante de substâncias psicotrópicas pode levar a dependência, múltiplos fatores podem estar envolvidos como condição econômica, meio social, desestruturação familiar, uso precoce, problemas psicológicos, entre outros (ALVAREZ S, et al., 2014).

Já na condição de dependência muitos usuários acabam perdendo seus empregos ou são abandonados por suas famílias, o que os levam a morar na rua. A precariedade de seu novo estilo de vida pode levá-los a danos irreparáveis a sua saúde física e mental (HINO P, et al, 2017).

A utilização de drogas por parte da população de rua é fruto da dependência adquirida antes de se tornarem moradores de rua, ou depois de já estarem na rua, em decorrência das dificuldades enfrentadas. $O$ uso de substâncias psicotrópicas representa não só a fuga temporária da realidade para eles, mas a tentativa de evitá-la (SPADONI L, et al., 2018).

O Vício na droga é algo incontrolável, que pode levar a consequências severas. A busca implacável pela droga leva muitos usuários a estados de saúde lamentáveis, a falta dela pode levá-los a crises graves de abstinências (MELO JR e MARCIEL SC, 2016).

A dependência em substâncias psicotrópicas é uma doença incurável que vai persistir mesmo frente a repercussões negativas, ou seja, ainda que diante de um quadro severo de crises ou doença causado pela dependência a pessoa tida como usuária tende a manter o vício. Higiene precária e estado nutricional deficitário é algo que está associado a essa condição (MAIA SDS, et al., 2018).

Um estudo realizado no centro de atenção psicossocial, em 2014, com 40 indivíduos, 20 entre eles 20 mulheres e 20 homens, que estavam em processo de tratamento da dependência química, observou-se que apenas $50 \%$ dos entrevistados faziam o uso da pasta dental, escova dental, e fio dental, e $42 \%$ relataram não fazer uso de nenhum componente. As drogas consumidas pelos dependentes são o crack (25\%), cocaína (25\%) e maconha (25\%), em seguida o álcool (22\%) (CIMA T e CORRÊA K, 2014).

O uso indiscriminado de álcool pode ocasionar irritamento na mucosa oral, está relacionado ao aumento de doenças como a gengivite, periodontites, cáries dentarias, lesões bucais malignas (MARQUES LARV, et al., 2015). A nicotina é uma droga presente nos cigarros e pode causar doenças gengivais, periodontite, cáries, perdas dentarias, halitose e risco de desenvolvimento de lesões malignas (MAIA SDS, et al., 2018).

A maconha é muito utilizada no Brasil, apesar de ser considera uma droga ilícita. O componente responsável por atuar no sistema nervo central é o canabidiol. Dependentes nessa droga podem apresentar cárie, doença periodontal, xerostomia, estomatite canábica, candidíase, lesões malignas (MARQUES LARV, et al., 2015).

Cocaína estimula o sistema nervoso central. Essa droga pode ser aspirada ou fumada como é o caso do crack que é um derivado da cocaína. O uso dessa substância e seus derivados pode ocasionar o aumento 
na ocorrência de alterações na cavidade bucal como cárie, erosão dentárias, doença periodontal, úlceras, candidíase oral e alterações nas células epiteliais. Essas alterações estão ligadas a diminuição do fluxo salivar, modificações no sistema imunológico e ao contato direto com a cavidade bucal (MELO CFD, et al., 2017).

A anfetamina é uma droga sintética que faz com que o cérebro trabalhe acima da sua função normal. A falta de sono e hiperatividade são efeitos ocasionados pelo consumo da substância. As pessoas que utilizam essa substância podem apresentar xerostomia, cáries rampantes, doença periodontal e bruxismo (MARQUES LARV, et al., 2015).

\section{A atuação dos profissionais da saúde bucal no programa Consultórios na Rua}

Com um olhar mais humanista o programa Consultório na Rua busca atuar promovendo, prevenindo e dando cuidados primários às pessoas que moram nas ruas (SOUZA VCA, et al, 2014).

As equipes são formadas por, no mínimo, quatro profissionais e podem ser compostas por médicos, enfermeiros, técnicos ou auxiliares de enfermagem, cirurgião-dentista, técnico em saúde bucal, psicólogos, assistentes sociais, terapeutas ocupacionais, agentes sociais, professor de educação física ou profissional com formação em arte e educação (BRASIL, 2017).

No Consultório na Rua os profissionais da saúde bucal deverão realizar tratamentos odontológicos, atividades educativas e lúdicas, dispensação de insumos de proteção à saúde, encaminhar e mediar o processo de encaminhamento para Rede de Saúde e intersetorial, acompanhar o cuidado das pessoas em situação de rua (BRASIL, 2011).

Neste contexto, o cirurgião dentista e o técnico em saúde bucal realizaram suas atividades em consultórios moveis ou nas unidades básicas de saúde em que estejam vinculados (BRASIL, 2011).

Dificuldades poderão estar presentes durante a prestação de serviço dos profissionais da saúde bucal. Indivíduos embriagados ou sobre o uso de outras drogas pode tornar o atendimento inesperado devido a imprevisibilidade dos atos devido ao uso dessas substâncias. Péssimas condições de higiene oral devido à falta de lugar e materiais como escova de dente, pasta e fio dental para realizar limpeza de sua boca pode acarretar em um tratamento não efetivo. As faltas constantes as consultas ou abandono ao tratamento também é um fator que pode gerar em empecilhos (MINISTERIO DA SAÚDE, 2012).

É de se ressaltar que um dos maiores problemas no tocante ao acesso ao SUS, ao controle e acompanhamento dessas pessoas, é que em sua maioria não possuem documentos de identificação (RG, CPF, Registro de nascimento, etc), documentos imprescindíveis para a elaboração do cartão do SUS (HALLAIS JAS e BARROS NF, 2015).

Um estudo realizado na cidade de São Paulo, no ano de 2016, em 4182 pessoas em situação de rua, observou que uma das principais necessidades foi a falta de prótese dentária. Devido ao atendimento realizado pelas equipes de saúde bucal percebeu-se um maior interesse no cadastramento no programa Consultório na Rua (CRUZ DSD, et al, 2017).

\section{A redução de danos fora da política nacional sobre drogas}

O Ministério da Saúde (2006) para garantir a liberdade de escolha dos indivíduos e tentar minimizar as consequências do consumo abusivo de álcool e outras drogas implementou a política de redução de danos sociais e a saúde. A política de redução de danos surgiu inicialmente no município de Santos-SP no ano de 1989, em decorrência dos altos números de pessoas contaminadas com HIV decorrente do compartilhamento de seringas entre usuários de drogas. Mas foi apenas em 2003 que as ações relacionadas a redução de danos deixaram de ser exclusivas dos programas de Doenças Sexualmente transmissíveis (DSTS) / Síndrome da imunodeficiência adquirida (AIDS) e se tornaram uma política do ministério da saúde para atenção integral de usuários de álcool e outras drogas (PASSOS EH e SOUZA TP, 2011).

O decreto n 9.761, de 11 de abril de 2019, já está em vigor e vale para todo território nacional, e traz como preceito objetivo a abstinência do dependente, não levando mais em conta a política de redução de danos, 
fato que poderá acarretar mudanças para os programas ligados a ela. A prevenção, tratamento, reinserção social, não discriminação, apoio, acolhimento aos dependentes foram preservados nessa política (BRASIL, 2019).

O programa Consultório na Rua estava ligado diretamente a política de redução de danos, com a entrada da nova Política Nacional sobre Drogas estabelecida pelo atual Governo, o programa poderá passar reformulações em suas diretrizes (BRASIL, 2019). Pode ser observado na literatura que a condição de morar na rua gera vulnerabilidade. Esses indivíduos estão submetidos a condições sub-humanas como a falta de comida, a ausência de local adequado para dormir e se higienizar, agressões constantes por parte até do próprio estado (MINISTERIO DA SAÚDE, 2014).

Um fator que se destacou e colabora para que muitas pessoas acabassem perdendo a capacidade de ser sustentar foi dependência em substâncias psicotrópicas. Muitos indivíduos relataram que a droga atua como uma válvula de escape da realidade (SPADONI L, et al., 2018).

O estudo realizado por Cima T e Corrêa K (2014), obteve resultados preocupantes acerca da higienização oral dos dependes químicos avaliados. Dos 40 indivíduos que participaram do estudo, $42 \%$ dos entrevistados relataram não realizar a escovação nem o uso do fio dental.

O consumo abusivo de drogas foi associado a alterações maléficas na cavidade bucal. Cárie dental, periodontites, xerostomia, halitose, perda de dentes, lesões teciduais podem ser observadas em indivíduos que se excedem na utilização dessas substâncias. Muitas dessas patologias se devem pelas péssimas condições de higiene oral dessas pessoas (MAIA SDS, et al., 2018).

O ministério da saúde divulga que a criação do programa Consultório na Rua, surgiu para atender as pessoas na condição de rua. A atuação dos profissionais da saúde bucal se dá de maneira a prevenir por meio de medidas educativas, realizar acompanhamentos, e tratamentos de baixa complexidade (BRASIL, 2011).

Souza VCA et al. (2014) em seu artigo fez menção ao fato de que aqueles moradores em situação de vulnerabilidade, por estarem morando nas ruas, não estão obrigados à adesão do programa, ou seja, não há nas diretrizes do programa Consultórios na Rua qualquer ponto que obrigue aos pacientes adesão ou a permanência no programa em testilha. Deve o programa prezar pela confiança que os profissionais devem perpassar aos pacientes.

Os profissionais da saúde bucal desempenham um papel fundamental dentro do programa como a realização de atendimentos, tratamentos de baixa complexidade, apresentações educacionais, e encaminhamentos para os centros de especialidades odontologia (BRASIL, 2011). Um estudo realizado por Cruz DSD, et al. (2017), em São Paulo, mostrou que muitas dessas pessoas necessitavam de prótese, fato que se deve as péssimas condições de higienes oral desses indivíduos. Além disso o programa teve uma maior adesão devido aos atendimentos odontológicos.

No manual sobre o cuidado à saúde junto a população em situação de rua trouxe as principais dificuldades enfrentadas pelos profissionais da saúde bucal e como eles poderão lidar durante seu vínculo com o programa. Pacientes sobre o efeito do uso de drogas, não colaboração com o tratamento, faltas frequentes as consultas e abandono do tratamento são alguns dos transtornos que os profissionais da odontologia deveram estar preparados para lidar (MINISTERIO DA SAÚDE, 2012).

Apesar de nova a atual política nacional sobre drogas, executada pelo governo em vigor, trouxe uma mudança importante que vai ocasionar uma possível mudança no programa. O governo brasileiro, em seu entendimento propôs por meio do decreto n.. 9.761, de 11 de abril de 2019, que a abstinência seria a melhor solução para os usuários. Por esse motivo a política de redução de danos não se enquadrou na política nacional sobre drogas (BRASIL, 2019).

A política de redução de danos cuida de pessoas que gostariam de sair do mundo das drogas e também dos indivíduos que desejam manter o vício, sempre de maneira a respeitar a vontade de cada cidadão (DIAS et al., 2014). 
A política de abstinência poderá gerar exclusão naqueles que desejarem manter seus hábitos deletérios, ou seja, cidadãos que já se encontram às margens sociais, serão excluídos até mesmo de programas sociais ligados a melhoria da sua qualidade de vida e manutenção de um bom estado de saúde (BRASIL, 2019).

\section{CONSIDERAÇÕES FINAIS}

Este trabalho possibilitou destacar a importância que os profissionais da saúde bucal desempenham no programa Consultório na Rua. Ressaltou que é com a participação deles que a odontologia alcança os moradores de rua, prestando um serviço de prevenção e tratamento daqueles que mais necessitam, cumprindo desse modo um dos principais princípios estabelecidos pelo SUS, a universalidade do atendimento.

\section{REFERÊNCIAS}

1- ALVAREZ $S$, et al. Causas da dependência química e suas consequências para o usuário e a família. Revista Enfermagem UFPE online, 2014; 8(3): 641-648.

2- BRASIL. 2011. In: Define as diretrizes de organização e funcionamento das Equipes de Consultório na Rua. Disponível em: bvsms.saude.gov.br/bvs/saudelequis/qm/2012/prt0122 2501 2012.html.Acesso em: 10 de mar. 2019.

3- BRASIL. 2015. In: Dispõe sobre a atualização do Anexo I, Listas de Substâncias Entorpecentes, Psicotrópicas, Precursoras e Outras sob Controle Especial, da Portaria SVS/MS no 344, de 12 de maio de 1998 e dá outras providências. Disponível em: www.lex.com.br/legis 26534834 RESOLUCAO N 8 DE 13 DE FEVEREIRO DE 2015.aspx.Acesso em: 6 de mar. 2019.

4- BRASIL. 2017. In: Em situação de rua. Disponível em: http://www.saude.gov.br/component/content/article/869politicas-de-equidade-em-saude/41381-em-. Acesso em:27 de out. 2019.

5- BRASIL. 2009. In: Pesquisa nacional sobre a população em situação de rua. Disponível em: www.mds.gov.br/webarquivos/publicacao/assistencia_social/Livros/Rua_aprendendo_a_contar.pdf.Acesso em: 14 de mar. 2019.

6- BRASIL. 2019. In: Política nacional sobre drogas. Disponível em: www.in.gov.br/materia/-/asset publisher/Kujrw0TZC2Mb/content/id/71137357/dole-2019-04-11-decreto-n-9-761-de-11-de-abril-de-2019-

71137316.Acesso em:14 de mar. 2019.

7- CARLINE E, et al. Livreto informativo sobre drogas psicotrópicas. 5nd ed. Brasília: Secretaria Nacional de Políticas sobre Drogas, 2011. 9-10 p.

8- CIMA T, CORRÊA K. Alterações bucais em dependentes químicos. Journal of Oral Investigation, 2014; 3(2): 8-12.

9- CRUZ DSV, et al. Atenção em saúde bucal para pessoas em situação de rua Um componente do consultório na rua. Secretaria Municipal da Saúde, 2017; 22(24): 1.

10- HALLIS JAS, BARROS NF. Consultório na Rua: visibilidades, invisibilidades e hipervisibilidade. Cadernos de saúde pública, 2015; 31(7): 1497-1504.

11- HINO P, et al. Pessoas que vivenciam situação de rua sob o olhar da saúde. Revista Brasileira de Enfermagem, 2019; 71(1): 732-740.

12- MAIA SDS, et al. Alterações bucais em usuários de drogas. Revista Esfera Acadêmica Saúde, 2016, 3(1): 1-17.

13- MARQUES LARV, et al. Abuso de drogas e suas consequências na saúde oral: uma revisão de literatura. Arquivo Brasileiro de Odontologia, 2015; 11(1): 26-31.

14- MELO JR, MARCIEL SC. Representação social do usuário de drogas na perspectiva de dependentes químicos. Psicologia: ciência e profissão, 2016; 36(1): 76-87.

15- MELO CFD, et al. MANIFESTAÇÕES ORAIS DE USUÁRIOS DE DROGAS ILÍCITAS: UMA REVISÃO DE LITERATURA NARRATIVA. Revista Ceuma Perspectivas, 2017; 29(1): 98-105.

16- MOREIRA JAC, PADILHA MI. ENTRE BATALHAS E PEDRAS: HISTÓRIAS DE VIDA DE MORADORES DE RUA, USUÁRIOS DE CRACK. Hacia la Promoción de la Salud, 2015; 20(1): 49-66.

17- MINISTERIO DA SAÚDE. Manual sobre à saúde junto a população em situação de rua. 1nd ed. Brasilia: Ministerio da Saúde, 2012. 76-77.

18- MINISTERIO DA SAÚDE. Saúde da população em situação de rua: Um direito humano. 1nd ed. Distrito Federal: Ministerio da Saúde, 2014. 26-27.

19- NONATO DM, RAIOL RWG. PESSOAS EM SITUAÇÃO DE RUA E VIOLÊNCIA: Entrelaçados em Nome da Suposta Garantia de Segurança Pública. Revista Direito em Debate, 2018; 49(1): 90-116. 
20- PAIVA IKSD, et al. Direito à saúde da população em situação de rua: reflexões sobre a problemática. Ciência e Saúde Coletiva, 2015; $21(8):$ 2595-2606.

21- PASSOS EH, SOUZA TP. Redução de danos e saúde pública: construções alternativas à política global de "guerra às drogas". Psicologia e Sociedade, 2018; 26(3): 285-291.

22- SOUZA VCA, et al. A experiência no serviço de Consultório de Rua na perspectiva dos profissionais: Contribuições para a atenção ao usuário de álcool e outras drogas. Cadernos Brasileiros de Terapia Ocupacional, 2014; $22(1)$ : 37 47.

23- SPADONI L, et al. Perfil de drogadição e práticas sociais entre moradores de rua. Psicologia e Saber Social, 2017; 6(1): 113-128.

24- UNITED NATIONS OFFICE ON DRUGS AND CRIME. 2018. World Drug Report. Disponível em: www.unodoc.org/wdr2018/index.html.Acesso em: 6 de mar. 